九州大学学術情報リポジトリ

Kyushu University Institutional Repository

Some considerations on the combination of estimates from different biological assays

Asano, Choichiro

Shionogi Pharamaceutical Co. I Kyushu University

https://doi.org/10.5109/12998

出版情報: 統計数理研究. 10 (1/2)，pp.17-32，1961-10. Research Association of Statistical Sciences

バージョン :

権利関係 : 


\title{
SOME CONSIDERATIONS ON THE COMBINATION OF ESTIMATES FROM DIFFERENT BIOLOGICAL ASSAYS
}

\author{
By \\ Chooichiro Asano \\ (Received January 25 and Reviced December 10, 1960)
}

\section{\$ 1. Introduction}

When we take aim to decide adequately an estimate of the potency of a test preparation by using the results of two or more biological assays conducted to data, whether these be assays of the same design and technique or assays by entirely different techniques, the problem of combining the estimates of the potency is encountered. This problem is often complicated with the various meaning of the estimate, particularly if the individual estimates were made by different workers using different procedures. Occasionally, the estimates to be combined may be considered that they must agree except for differences attributable to sampling error. On the other hand, however, they may have not necessarily the agreement as a general rule in the sense of that the duty of combining the estimates discharges some moderate adjustment of the potency of a test preparation on the basis of a physical ground.

In either case we are now in a situation to make a best composite figure as the potency of a test preparation. Then in the formation of the combined estimate, most importance will be attached to the individual values that are most precise or reliable, and some type of weighted mean will therefore be wanted very often.

The problem of making a combined estimate of the weighted mean type has been discussed previously by many authors, that is, Cochran [1], [2], Yates and Cochran [1], Bliss [1], Finney [1] and et al. And the purpose of this paper in treating the subject again is to discuss it from the viewpoint of the recent successive process of statistical inferences based on a scheme of Bancroft [1], Kitagawa [1], [2], Bennet [1], Huntsberger [1] and et $a l$. and attempts to give some results and recommendations for the biological research workers in practice.

In conclusion the author wishes his hearty thanks to Prof. ' $\Gamma$. Kitagawa for his kind suggestions and encouragement. 


\section{§. The inferences using the results of two biological assays with known variances}

Let $O_{n_{1}}:\left(x_{11}, x_{12}, \ldots, x_{1 n_{1}}\right)$ be a random sample of $n_{1}$ from a normal population $N\left(\mu_{1}, \sigma_{1}^{2}\right)$ and let $O_{n 2}:\left(x_{21}, x_{22}, \ldots, x_{2 n_{2}}\right)$ be a random sample of $n_{2}$ from another normal population $N\left(\mu_{2}, \sigma_{2}^{2}\right)$. The values of these two population variances are known to us and they are distinct each other, but the populations have not necessarily the same population mean. Then our final aim is to estimate a value of $\left(\mu_{1}+\mu_{2}\right) / 2$ with the best precision.

\subsection{Preliminary testing procedure (1)}

We are now concerned with the rule of the statistical procedure formulated in the following way:

(i) Let $\bar{x}_{i}$ and $\sigma_{i}^{2}$ be the sample mean and the population variance defined by the $i$-th population, $(i=1,2)$.

(ii) Let the statistic $d$ be defined by $d=\left|\bar{x}_{1}-\bar{x}_{2}\right| / \sqrt{\frac{\sigma_{1}^{2}}{n_{1}}+\frac{\sigma_{2}^{2}}{n_{2}}}$.

(iii) Let us define the statistic $\overline{\bar{x}}$ in the following way:

$$
\begin{aligned}
& \text { (a) } \overline{\bar{x}}=\left(\frac{n_{1} \bar{x}_{1}}{\sigma_{1}^{2}}+\frac{n_{2} \bar{x}_{2}}{\sigma_{2}^{2}}\right) /\left(\frac{n_{1}}{\sigma_{1}^{2}}+\frac{n_{2}}{\sigma_{2}^{2}}\right), \text { if } d \leq \Lambda, \\
& \text { (b) } \bar{x}=\left(\bar{x}_{1}+\bar{x}_{2}\right) / 2, \text { if } d>\Lambda,
\end{aligned}
$$

where the prescribed constant $A$ is corresponding to a value of the $\alpha_{\Lambda^{-}}$ percent point of the normal distribution $N(0,1)$.

The necessity for such inference procedure is based upon the following motivation. Under the situation where the assumption $\mu_{1}=\mu_{2}=\mu$ to be preliminary tested holds true, the estimate with weights inversely proportional to the population variances which gives us the best estimate of common $\mu$ is known to be prefered. On the other hand, however, under the situation where the assumption is naied by the preliminary test, a simplest estimate $\left(\bar{x}_{1}+\bar{x}_{2}\right) / 2$ for $\left(\mu_{1}+\mu_{2}\right) / 2$ may be quite reasonable because of that the estimate gives us the unbiassed estimate.

Thus our inference procedure is divided into two branches for the sake of aiming at the improvement of the precision of estimate and is distinct essentially from the theories of combined estimate of weighted mean type discussed previously. The results of our inference procedure is obtained as follows.

Theorem 1.1. The distribution function of $\overline{\bar{x}}$ is given by

$$
\begin{aligned}
\operatorname{Pr} .\{\overline{\bar{x}}<u\} & =\left\{\Phi\left(\Lambda-m_{X}\right)-\Phi\left(-\Lambda-m_{X}\right)\right\} \Phi\left(\frac{u-m_{Y}}{\sigma_{Y}}\right) \\
& +\frac{1}{\sqrt{2 \pi}} \int_{-\infty}^{\frac{u-m_{Z}}{\sigma_{Z}}}\left\{1-\Phi\left(\frac{\Lambda-m_{X}-\rho_{X Z} t}{\sqrt{1-\rho_{X Z}^{2}}}\right)-\Phi\left(\frac{-\Lambda-m_{X}-\rho_{X Z} t}{\sqrt{1-\rho_{X Z}^{2}}}\right)\right\} e^{-t / 2 / 2} d t,
\end{aligned}
$$


where we put

$$
\begin{aligned}
& m_{X}=\left(\mu_{1}-\mu_{2}\right) / \sqrt{\frac{\sigma_{1}^{2}}{n_{1}}+\frac{\sigma_{2}^{2}}{n_{2}}}, m_{Y}=\left(\frac{n_{1}}{\sigma_{1}^{2}} \mu_{1}+\frac{n_{2}}{\sigma_{2}^{2}} \mu_{2}\right)\left(\frac{n_{1}}{\sigma_{1}^{2}}+\frac{n_{2}}{\sigma_{2}^{2}}\right), m_{Z}=\left(\mu_{1}+\mu_{2}\right) / 2, \\
& \sigma_{Y}^{2}=\left(\frac{n_{1}}{\sigma_{1}^{2}}+\frac{n_{2}}{\sigma_{2}^{2}}\right)^{-1}, \sigma_{Z}^{2}=\left(\frac{\sigma_{1}^{2}}{n_{1}}+\frac{\sigma_{2}^{2}}{n_{2}}\right) / 4 \quad \text { and } \quad \rho_{X Z}=\left(\frac{\sigma_{1}^{2}}{n_{1}}-\frac{\sigma_{2}^{2}}{n_{2}}\right) /\left(\frac{\sigma_{1}^{2}}{n_{1}}+\frac{\sigma_{2}^{2}}{n_{2}}\right) .
\end{aligned}
$$

Proof: Let us put

$$
\operatorname{Pr} .\{\bar{x}<u\}=\operatorname{Pr}\left\{\left(\frac{n_{1} \bar{x}_{1}}{\sigma_{1}^{2}}+\frac{n_{2} \bar{x}_{2}}{\sigma_{2}^{2}}\right)\left(\frac{n_{1}}{\sigma_{1}^{2}}+\frac{n_{2}}{\sigma_{2}^{2}}\right)^{-1}<u, D_{1}\right\}+\operatorname{Pr}\left\{\frac{\bar{x}_{1}+\bar{x}_{2}}{2}<u, D_{2}\right\},
$$

where $D_{1}$ and $D_{2}$ are the domains in the sample space $\left(x_{11}, \ldots, x_{1 n_{1}}, x_{21}, \ldots\right.$, $x_{2 n_{2}}$ ) defined by the above relations (a) and (b) respectively. Then, defining $X, Y$ and $Z$ as $\left(\bar{x}_{1}-\bar{x}_{2}\right)\left(\frac{\sigma_{1}^{2}}{n_{1}}+\frac{\sigma_{2}^{2}}{n_{2}}\right)^{-1 / 2},\left(\frac{n_{1}}{\sigma_{1}^{2}} \bar{x}_{1}+\frac{n_{2}}{\sigma_{2}^{2}} \bar{x}_{2}\right)\left(\frac{n_{1}}{\sigma_{1}^{2}}+\frac{n_{2}}{\sigma_{2}^{2}}\right)^{-1}$ and $\left(\bar{x}_{1}+\bar{x}_{2}\right) / 2$ respectively, we may obtain that

$$
\begin{array}{r}
\operatorname{Pr} .\left\{Y<u, D_{1}\right\}=\left(2 \pi \sigma_{X} \sigma_{Y}\right)^{-1} \int_{-\infty}^{u} \int_{-\Lambda}^{\Lambda} \exp \left[-\frac{1}{2}\left\{\frac{\left(X-m_{X}\right)^{2}}{\sigma_{X}^{2}}+\frac{\left(Y-m_{Y\rangle}^{\prime 2}\right.}{\sigma_{Y}^{2}}\right\}\right] d X d X, \\
\operatorname{Pr} .\left\{Z<u, D_{2}\right\}=\left\{2 \pi \sigma_{X} \sigma_{Z}\left(1-\rho_{X Z}^{2}\right)\right\}^{-1} \int_{-\infty}^{u} \int_{\Lambda<|X|<\infty} \exp \left[-\frac{1}{2\left(1-\rho_{X Z}^{2}\right)}\left\{\frac{\left(X-m_{X}\right)^{2}}{\sigma_{X}^{2}}\right.\right. \\
\left.\left.-2 \rho_{X Z} \frac{\left(X-m_{X}\right)\left(Z-m_{Z}\right)}{\sigma_{X} \sigma_{Z}}+\frac{\left(Z-m_{Z}\right)^{2}}{\sigma_{Z}^{2}}\right\}\right] d X d Z,
\end{array}
$$

where $X$ is independent of $Y$ and $m_{X}, m_{Y}, m_{Z}, \sigma_{Y}, \sigma_{z}$ and $\rho_{X Z}$ are given by (2.1.2) and $\sigma_{X}^{2}=1$. Thus we may obtain easily (2.1.1).

Theorem 1.2. The mean value $E\{\overline{\bar{x}}\}$ and the mean square deviation $E\left\{\left(\overline{\bar{x}}-\frac{\mu_{1}+\mu_{2}}{2}\right)^{2}\right\}$ of the estimate $\overline{\bar{x}}$ are given by

$$
\begin{aligned}
E\{\bar{x}\}=m_{Z}+m_{Y}\left\{\Phi\left(\Lambda-m_{X}\right)-\Phi\left(-\Lambda-m_{X}\right)\right\}-\frac{1}{\sqrt{2 \pi}} \int_{-\infty}^{\infty}\left(m_{Z}+\sigma_{Z} t\right) \\
\cdot\left\{\Phi\left(\frac{\Lambda-m_{X}-\rho_{X Z} t}{\sqrt{1-\rho_{X Z}^{2}}}\right)-\Phi\left(\frac{-\Lambda-m_{X}-\rho_{X Z} t}{\sqrt{1-\rho_{X Z}^{2}}}\right)\right\} e^{-t: / 2} d t,
\end{aligned}
$$

and

$$
\begin{aligned}
& \text { M.S.D. }\{\overline{\boldsymbol{x}}\}=\sigma_{Z}^{2}+\left(m_{Y}^{2}-2 m_{Y} m_{Z}+\sigma_{Y}^{2}\right)\left\{\Phi\left(\Lambda-m_{X}\right)-\Phi\left(-\Lambda-m_{X}\right)\right\} \\
& +\frac{1}{\sqrt{2}} \int_{-\infty}^{\infty}\left(m_{Z}^{2}-t^{2} \sigma_{Z}^{2}\right)\left\{\Phi\left(\frac{\Lambda-m_{X}-\rho_{X Z} t}{\sqrt{1-\rho_{X Z}^{2}}}\right)-\Phi\left(\frac{-\Lambda-m_{X}-\rho_{X Z Z} t}{\sqrt{1-\rho_{X Z}^{2}}}\right)\right\} e^{-t 2 / 2} d t .
\end{aligned}
$$

Proof: The mean value (2.1.5) is evident, and the mean square deviation is obtained by 
(2.1.7) $E\left\{\overline{\bar{x}}^{2}\right\}=\left(m_{Y}^{2}+\sigma_{Y}^{2}\right)\left\{\Phi\left(\Lambda-m_{X}\right)-\Phi\left(-\Lambda-m_{X}\right)\right\}+m_{Z}^{2}+\sigma_{z}^{2}-\frac{1}{\sqrt{2 \pi}} \int_{-\infty}^{\infty}\left(m_{z}+\sigma_{z} t\right)^{2}$

$$
\cdot\left\{\Phi\left(\frac{\Lambda-m_{X}-\rho_{X X} t}{\sqrt{1-\rho_{X Z}^{2}}}\right)-\Phi\left(\frac{-\Lambda-m_{X}-\rho_{X, 2} t}{\sqrt{1-\rho_{X Z}^{2}}}\right)\right\} e^{-t^{2 / 2}} d t .
$$

Corollary 1.1. Specially when $\mu_{1}=\mu_{2}=\mu, \bar{x}$ is an unbiassed estimate of $\mu$ and M.S.D. $\{\bar{x}\}$ becomes as follows:

$$
\text { M.S.D. }\{\overline{\bar{x}}\}=\frac{1}{4}\left(\frac{\sigma_{1}^{2}}{n_{1}}+\frac{\sigma_{2}^{2}}{n_{2}}\right)-\frac{1}{4} \frac{n_{1} n_{2}}{\sigma_{1}^{2} \sigma_{2}^{2}}\left(\frac{\sigma_{1}^{2}}{n_{1}}-\frac{\sigma_{2}^{2}}{n_{2}}\right)^{2}\{\Phi(\Lambda)-\Phi(-\Lambda)\} .
$$

Of course, in this special case, we may find easily that taking zero as a value of the $A$ is prefered for minimizing the M.S.D. $\{\bar{x}\}$.

Corollary 1.2. Specially when $\sigma_{1}^{2}=\sigma_{2}^{2}=\sigma^{2}$, the values of $E\{\bar{x}\}$ and M.S.D. $\{\overline{\bar{x}}\}$ are shown as follows:

$$
\begin{gathered}
\text { (2.1.9) } E\{\bar{x}\}=\frac{\mu_{1}+\mu_{2}}{2}+\frac{n_{1} \mu_{1}+n_{2} \mu_{2}}{n_{1}+n_{2}}\left\{\Phi\left(\Lambda-\frac{\sqrt{n_{1} n_{2}}\left(\mu_{1}-\mu_{2}\right)}{\sqrt{n_{1}+n_{2}} \sigma}\right)\right. \\
\left.-\Phi\left(-\Lambda-\frac{\sqrt{n_{1} n_{2}}\left(\mu_{1}-\mu_{2}\right)}{\sqrt{n_{1}+n_{2}} \sigma}\right)\right\}-\frac{1}{\sqrt{2 \pi}} \int_{-\infty}^{\infty}\left(\frac{\mu_{1}+\mu_{2}}{2}+\frac{\sqrt{n_{1}+n_{2}}}{2 \sqrt{n_{1} n_{2}}} \sigma t\right) \\
\cdot\left\{\Phi\left(\frac{\Lambda-\frac{\sqrt{n_{1} n_{2}}\left(\mu_{1}-\mu_{2}\right)}{\sqrt{n_{1}+n_{2}} \sigma}+\frac{n_{1}-n_{2}}{n_{1}+n_{2}} t}{2 \sqrt{n_{1} n_{2}} /\left(n_{1}+n_{2}\right)}\right)-\Phi\left(\frac{-\Lambda-\frac{\sqrt{n_{1} n_{2}}\left(\mu_{1}-\mu_{2}\right)}{\sqrt{n_{1}+n_{2}} \sigma}+\frac{n_{1}-n_{2}}{n_{1}+n_{2}} t}{2 \sqrt{n_{1} n_{2}} /\left(n_{1}+n_{2}\right)}\right)\right\} e^{-t 2 / 2} d t,
\end{gathered}
$$

and

$$
\begin{array}{r}
\text { 10) M.S.D. }\{\overline{\bar{x}}\}=\frac{\left(n_{1}+n_{2}\right) \sigma^{2}}{4 n_{1} n_{2}}+\left\{\frac{\sigma^{4}}{\left(n_{1}+n_{2}\right)^{2}}-\frac{\left(n_{1} \mu_{1}+n_{2} \mu_{2}\right)\left(n_{2} \mu_{1}+n_{1} \mu_{2}\right)}{\left(n_{1}+n_{2}\right)^{2}}\right\} \\
\cdot\left\{\Phi\left(\Lambda-\frac{\sqrt{n_{1} n_{2}}\left(\mu_{1}-\mu_{2}\right)}{\sqrt{\prime} \overline{n_{1}+n_{2}} \sigma}\right)-\Phi\left(-\Lambda-\frac{\sqrt{n_{1} n_{2}}\left(\mu_{1}-\mu_{2}\right)}{\sqrt{n_{1}+n_{2}} \sigma}\right)\right\}+\frac{1}{\sqrt{2 \pi}} \int\left(\frac{\left(\mu_{1}+\mu_{2}\right)^{2}}{4}\right. \\
\left.-\frac{\left(n_{1}+n_{2}\right) \sigma^{2}}{4 n_{1} n_{2}} t^{2}\right)
\end{array}
$$

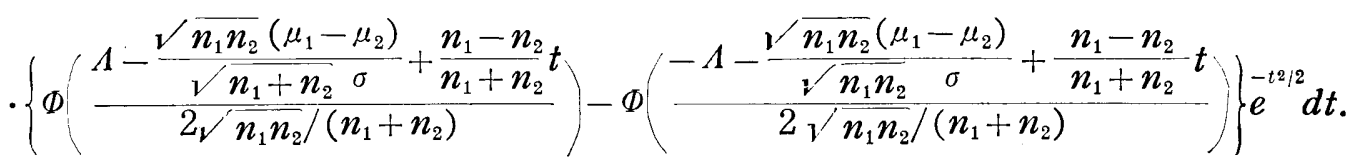

It may be noted in this special case that the inference procedure defined by $\Lambda=0$ gives us the unbiassed estimate but does not always show a minimum value of the M.S.D. $\{\bar{x}\}$.

\subsection{On an examination in view of a weighting procedure}

Let us consider a weighting procedure which uses weights determined 
immediately by the observed value of the preliminary test function. That is to say, for an estimator of $\left(\mu_{1}+\mu_{2}\right) / 2$, a function in the present weighting procedure is defined by

$$
W(T)=\frac{\bar{x}_{1}+\bar{x}_{2}}{2} \phi(T)+\frac{\frac{n_{1} \bar{x}_{1}}{\sigma_{1}^{2}}+\frac{n_{2} \bar{x}_{2}}{\sigma_{2}^{2}}}{\frac{n_{1}}{\sigma_{1}^{2}}+\frac{n_{2}}{\sigma_{2}^{2}}}\{1-\phi(T)\},
$$

where $T$ denotes the statistic which will provide the test of the hypothesis that $\mu_{1}=\mu_{2}$ against the class of alternatives $\mu_{1} \neq \mu_{2}$ and $\phi(T)$ is a value of the test function and denotes the weighting function in weighting procedure at the same time. If $\phi(T)$ is defined as (i) $\phi(T)=0$ in the case $T \subset A_{\alpha}$ and (ii) $\phi(T)=1$ in the case $T \subset R_{\alpha}$, where $A_{\alpha}$ and $R_{\alpha}$ are the acceptance and rejection regions for the test of $H$ with probability of the first type error equals to $\alpha$, then $W(T)$ reduces to our estimator in sometimes pooling procedure based upon the preliminary test of significance. In order to determine whether or not $W(T)$ offers any advantages over the results of the preliminary test procedure, the mean square error about the true value of $\left(\mu_{1}+\mu_{2}\right) / 2$ is used as a criterion of goodness.

Now let us consider an estimate which is substituted a constant $A$ for $\phi(T)$ in (2.2.1). Then the mean square deviate about $\left(\mu_{1}+\mu_{2}\right) / 2$ is obtained as follows :

$$
V\{W(A)\}=\frac{\left(\frac{\sigma_{1}^{2}}{n_{1}}-\frac{\sigma_{2}^{2}}{n_{2}}\right)^{2}}{4\left(\frac{\sigma_{1}^{2}}{n_{1}}+\frac{\sigma_{2}^{2}}{n_{2}}\right)}\left\{\frac{4 \frac{\sigma_{1}^{2} \sigma_{2}^{2}}{n_{1} n_{2}}}{\left(\frac{\sigma_{1}^{2}}{n_{1}}-\frac{\sigma_{2}^{2}}{n_{2}}\right)^{2}}+A^{2}+(1-A)^{2}\left(\frac{\mu_{1}-\mu_{2}}{\sqrt{\frac{\sigma_{1}^{2}}{n_{1}}+\frac{\sigma_{2}^{2}}{n_{2}}}}\right)^{2}\right\},
$$

and this is minimized in case when $A=\left(\mu_{1}-\mu_{2}\right)^{2} /\left\{\left(\frac{\sigma_{1}^{2}}{n_{1}}+\frac{\sigma_{2}^{2}}{n_{2}}\right)+\left(\mu_{1}-\mu_{2}\right)^{2}\right\}$.

From the view-point of (2.2.2) a criterion for the choice of the value of $A$ may be easily considered with a certain rationality as follows:

$$
\left\{\begin{array}{cccc}
A=0, & \text { if } & \left(\mu_{1}-\mu_{2}\right)^{2} /\left(\frac{\sigma_{1}^{2}}{n_{1}}+\frac{\sigma_{2}^{2}}{n_{2}}\right) & <1, \\
A=1 / 2, & \text { if } & \prime \prime & =1, \\
A=1, & \text { if } & \prime \prime & >1,
\end{array}\right.
$$

and this will be applied to the preliminary testing procedure (2) in section 2.3.

In the present weighting procedure, since the value of $\left(\mu_{1}-\mu_{2}\right) / \sqrt{\frac{\sigma_{1}^{2}}{n_{1}}+\frac{\sigma_{2}^{2}}{n_{2}}}$ is unknown to us, we may and now shall substitute a statistic $T$ defined by $\left(\bar{x}_{1}-\bar{x}_{2}\right) / \sqrt{\frac{\sigma_{1}^{2}}{n_{1}}+\frac{\sigma_{2}^{2}}{n_{2}}}$ for the parameter and further let $\phi(T)$ in (2.2.1) be $\phi_{0}(T)$ defined by $T^{2} /\left(1+T^{2}\right)$. Then we may obtain a following theorem 
corresponding to Huntsberger [1].

Theorem 2.1. The expectation and the mean square deviation of the estimator $W_{0}(T)$ given by the weighting procedure (2.2.1) is obtained as follows:

$$
E\left\{W_{0}(T)\right\}=\frac{\mu_{1}+\mu_{2}}{2}+\frac{\frac{n_{1}}{\sigma_{1}^{2}}-\frac{n_{2}}{\sigma_{2}^{2}}}{2 \sqrt{\frac{\boldsymbol{n}_{1}}{\sigma_{1}^{2}}+\frac{n_{2}}{\sigma_{2}^{2}}}} \gamma\left\{\sqrt{\frac{\sigma_{1}^{2} \sigma_{2}^{2}}{n_{1} n_{2}}}-1+\sum_{i=0}^{\infty} \frac{\mathrm{e}^{-\gamma^{2} / 2} \gamma^{2 i}}{2^{2} i !}-A_{i+1}\right\},
$$

and

$$
\begin{aligned}
& \text { M.S.D. }\left\{W_{0}(T)\right\} \equiv E\left\{\left(W_{0}(T)-\frac{\mu_{1}-\mu_{2}}{2}\right)^{2}\right\} \\
& \quad=I(\gamma)-r^{2}\left(1-\frac{\sigma_{1}^{2} \sigma_{2}^{2}}{n_{1} n_{2}}\right)-2 r^{2}\left(1-\sqrt{\frac{\sigma_{1}^{2} \sigma_{2}^{2}}{n_{1} n_{2}}}\right) \sum_{i=0}^{\infty} \frac{e^{-\gamma^{2 / 2}} r^{2 l}}{2^{i} i !} A_{i+1},
\end{aligned}
$$

where we put like in Huntsberger [1] that $I(\gamma)=1+3 H(\gamma)-5 G(\gamma)$,

$$
\begin{aligned}
& H(r)=\sum_{i=0}^{\infty} \frac{e^{-\gamma^{2} / 2}}{i !}\left(\frac{\gamma^{2}}{2}\right)^{i} A_{i}, G(r)=\frac{e^{-\gamma^{2} / 2}}{2}\left\{1+\sum_{i=1}^{\infty} \frac{r}{2^{i} i !}\left(A_{i-1}-A_{i}\right)\right\}, \\
& A_{0}=e^{1 / 2} \int_{1}^{\infty} e^{-x^{2} / 2} d x, \quad A_{i}=\left(1-A_{i}-1\right) /(2 i-1)=e^{1 / 2} \int_{1}^{\infty} x^{-2 i} e^{-x^{2} / 2} d x .
\end{aligned}
$$

In order to determine whether or not $W_{0}(T)$ offers any advantage over the previous inference procedure given at section 2.1 as an estimation of $\left(\mu_{1}+\mu_{2}\right) / 2$, the mean square deviation about the true parameter value is used as a criterion of goodness. It seems to us, however, that $W_{0}(T)$ has not so goodness from the conclusions of Huntsberger [1] and Asano-Sugimura [1] and here we may be confined within a notion (2.2.3).

\subsection{Preliminary testing procedure (2)}

From the view-point of the criterion for the choice of the value of $A$ in section 2.2, we may and now shall propose another preliminary testing procedure which is distinct from section 2.1 in order to aim at the improvement of the precision of the estimate of $\left(\mu_{1}+\mu_{2}\right) / 2$. The formation proposed here is concerned with one of the preliminary two-sided testing procedure which were descrived previously with the fundamental aspects by the author [1].

The rule of our statistical procedure is now specialized under the same notation as those of section 2.1 in the following way:

(i) Let the statistic $d$ be defined by $d=\left(\bar{x}_{1}-\bar{x}_{2}\right) / \sqrt{\frac{\sigma_{1}^{2}}{n_{1}}+\frac{\sigma_{2}^{2}}{n_{2}}}$,

(ii) Let us define the statistic $\overline{\bar{x}}$ in the following way: 
(a) $\overline{\bar{x}}=\left(\frac{n_{1} \bar{x}_{1}}{\sigma_{1}^{2}}+\frac{n_{2} \bar{x}_{2}}{\sigma_{2}^{2}}\right) /\left(\frac{n_{1}}{\sigma_{1}^{2}}+\frac{n_{2}}{\sigma_{2}^{2}}\right)$, if $d_{2}<\lambda$,

(b) $\overline{\bar{x}}=\frac{1}{2}\left\{\frac{\frac{n_{1} \bar{x}_{1}}{\sigma_{1}^{2}}+\frac{n_{2} \bar{x}_{2}}{\sigma_{2}^{2}}}{\frac{n_{1}}{\sigma_{1}^{2}}+\frac{n_{2}}{\sigma_{2}^{2}}}+\frac{\bar{x}_{1}+\bar{x}_{2}}{2}\right\}$, if $\lambda \leq d^{2} \leq A$,

(c) $\overline{\bar{x}}=\left(\bar{x}_{1}+\bar{x}_{2}\right) / 2$, if $d_{2}<\Lambda$,

where the switching constants $A$ and $\lambda$ are prescribed constants.

We are now going some results on the mean and the mean square deviation of the estimate given by the present modified inference procedure.

Theorem 2.2 The distribution function of $\bar{x}$ is given by

$$
\operatorname{Pr} .\{\bar{x}<u\}=\left[\int_{D(\mathrm{I})}+\iint_{D(\mathrm{~b})}+\iint_{D(\mathrm{c})}\right] f(z, y) d z d y,
$$

where $D_{(\mathrm{a})}, D_{(\mathrm{b})}$ and $D_{(\mathrm{c})}$ are the domains in the sample space defined by the procedure (a), (b) and (c) respectively and are denoted by

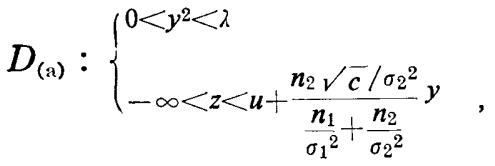

$$
\begin{aligned}
& D_{(\mathrm{b})}:\left\{\begin{array}{l}
\lambda \leq y^{2} \leq \Lambda \\
-\infty<z<u+\left(1-\frac{\frac{n_{1}}{\sigma_{1}{ }^{2}}-\frac{n_{2}}{\sigma_{2}{ }^{2}}}{2\left(\frac{n_{1}}{\sigma_{1}{ }^{2}}+\frac{n_{2}}{\sigma_{2}{ }^{2}}\right)}\right) \frac{\sqrt{c}}{2} y,
\end{array}\right.
\end{aligned}
$$

and

$$
D_{(\mathrm{c})}:\left\{\begin{array}{l}
1<y^{2}<\infty \\
-\infty<z<u+\frac{\sqrt{c}}{2} y,
\end{array},\right.
$$

and where we put

$$
f(z, y)=-\frac{\sqrt{n_{1} n_{2} c}}{2 \pi \sigma_{1} \sigma_{2}} \exp \left[-\frac{n_{1}\left(z-\mu_{1}\right)^{2}}{2 \sigma_{1}^{2}}-\frac{n_{2}\left(z-\sqrt{c} y-\mu_{2}\right)^{2}}{2 \sigma_{2}^{2}}\right], c=\frac{\sigma_{1}^{2}}{n_{1}}+\frac{\sigma_{2}^{2}}{n_{2}}
$$

aud the switching constants $\lambda$ and $\Lambda$ are denoted by $\left(\sqrt{\lambda^{\prime}}+1\right)^{2}$ and $\left(\sqrt{ } / \overline{\Lambda^{\prime}}+1\right)^{2}$ in terms of some percent points $\lambda^{\prime}$ and $\Lambda^{\prime}$ of $N(0,1)$ respectively.

Proof: We may easily obtain by transforming the joint distribution of $\bar{x}_{1}$ and independent $\bar{x}_{2}$ to that of $y$ and $z$ defined afresh by $\left(\bar{x}_{1}-\bar{x}_{2}\right) / \sqrt{c}$ and $\bar{x}_{1}$ and from being a preliminary testing hypothesis $H_{0}:\left(\mu_{1}-\mu_{2}\right) / \sqrt{ } / \bar{c}=1$.

Theorem 2.3. The mean value $E\{\overline{\bar{x}}\}$ and the mean square deviation $E\left\{\left(\bar{x}-\left(\mu_{1}+\mu_{2}\right) / 2\right)^{2}\right\}$ of the estimate $\overline{\bar{x}}$ are given by

(2.3.4.) $E\{\overline{\bar{x}}\}=\frac{\mu_{1}+\mu_{2}}{2}-\frac{1}{\sqrt{2 \pi}} \int_{-\infty}^{\infty} e^{-t 2 / 2}\left[\left(\frac{\mu_{1}+\mu_{2}}{2}+\frac{\sigma_{1}}{2 \sqrt{\boldsymbol{n}_{1}}} t\right)\left\{\Phi^{(0)}\left(\frac{\mu_{1}-\mu_{2}+\sqrt{\boldsymbol{c} \boldsymbol{A}}+t \sigma_{1} / \sqrt{\boldsymbol{n}_{1}}}{\sigma_{2} / \sqrt{\boldsymbol{n}_{2}}}\right)\right.\right.$

$$
\left.-\Phi^{(0)}\left(\frac{\mu_{1}-\mu_{2}-\sqrt{\boldsymbol{c} \Lambda}+t \sigma_{1} / \sqrt{\boldsymbol{n}_{1}}}{\sigma_{2} / \sqrt{\boldsymbol{n}_{2}}}\right)\right\}+\frac{\sigma_{2}}{2 \sqrt{\boldsymbol{n}_{2}}}\left\{\Phi^{(1)}\left(\frac{\mu_{1}-\mu_{2}+\sqrt{\overline{c \Lambda}}+t \sigma_{1} / \sqrt{\boldsymbol{n}_{1}}}{\sigma_{2} / \sqrt{\boldsymbol{n}_{2}}}\right)\right.
$$




$$
\begin{aligned}
& \left.\left.-\Phi^{(1)}\left(\frac{\mu_{1}-\mu_{2}-\sqrt{c \Lambda}+t \sigma_{1} / \sqrt{n_{1}}}{\sigma_{2} / \sqrt{n_{2}}}\right)\right\}\right] d t \\
& +\frac{1}{\sqrt{2 \pi}} \int_{-\infty}^{\infty} e^{-t 2 / 2}\left[( \frac { \frac { \boldsymbol { n } _ { 1 } } { \sigma _ { 1 } ^ { 2 } } \mu _ { 1 } + \frac { \boldsymbol { n } _ { 2 } } { \sigma _ { 2 } ^ { 2 } } \mu _ { 2 } } { \frac { \boldsymbol { n } _ { 1 } } { \sigma _ { 1 } ^ { 2 } } + \frac { \boldsymbol { n } _ { 2 } } { \sigma _ { 2 } ^ { 2 } } } + \frac { t _ { 1 } / \overline { n _ { 1 } } / \sigma _ { 1 } } { \frac { \boldsymbol { n } _ { 1 } } { \sigma _ { 1 } ^ { 2 } } + \frac { \boldsymbol { n } _ { 2 } } { \sigma _ { 2 } ^ { 2 } } } ) \left\{\Phi^{(0)}\left(\frac{\mu_{1}-\mu_{2}+\sqrt{\boldsymbol{c} \lambda}+t \sigma_{1} / \sqrt{\boldsymbol{n}_{1}}}{\sigma_{2} / \sqrt{\boldsymbol{n}_{2}}}\right)\right.\right. \\
& \left.-\Phi^{(0)}\left(\frac{\mu_{1}-\mu_{2}-\sqrt{c \lambda}+t \sigma_{1} / \sqrt{\boldsymbol{n}_{1}}}{\sigma_{2} / \sqrt{\boldsymbol{n}_{2}}}\right)\right\}+\frac{\sqrt{\boldsymbol{n}_{2}} / \sigma_{2}}{\frac{\boldsymbol{n}_{1}}{\sigma_{1}^{2}}+\frac{\boldsymbol{n}_{2}}{\sigma_{2}^{2}}}\left\{\Phi^{(1)}\left(\frac{\mu_{1}-\mu_{2}+\sqrt{c \bar{\lambda}}+t \sigma_{1} / \sqrt{\boldsymbol{n}_{1}}}{\sigma_{2} / \sqrt{\boldsymbol{n}_{2}}}\right)\right. \\
& \left.\left.-\Phi^{(1)}\left(\frac{\mu_{1}-\mu_{2}-\sqrt{\boldsymbol{c} \lambda}+t \sigma_{1} / \sqrt{\boldsymbol{n}_{1}}}{\sigma_{2} / \sqrt{\boldsymbol{n}_{2}}}\right)\right\}\right] d t \\
& +\frac{1}{4 / 2 \pi}\left(\frac{n_{1}}{\sigma_{1}^{2}}+\frac{n_{2}}{\sigma_{2}^{2}}\right)^{-1} \int_{-\infty}^{\infty} e^{-t 2 / 2}\left[\left\{\left(\frac{3 n_{1}}{\sigma_{1}^{2}}+\frac{n_{2}}{\sigma_{2}^{2}}\right) \mu_{1}+\left(\frac{3 n_{2}}{\sigma_{2}^{2}}+\frac{n_{1}}{\sigma_{1}^{2}}\right) \mu_{2}+\left(\frac{3 n_{1}}{\sigma_{1}^{2}}+\frac{n_{2}}{\sigma_{2}^{2}}\right) \frac{\sigma_{1}}{\sqrt{n_{1}}} t\right\}\right. \\
& \cdot\left\{\Phi^{(0)}\left(\frac{\mu_{1}-\mu_{2}-\sqrt{c \lambda}+t \sigma_{1} / \sqrt{n_{1}}}{\sigma_{2} / \sqrt{n_{2}}}\right)-\Phi^{(0)} \cdot\left(\frac{\mu_{1}-\mu_{2}-\sqrt{c \Lambda}+t \sigma_{1} / \sqrt{n_{1}}}{\sigma_{2} / \sqrt{n_{2}}}\right)\right. \\
& \left.+\Phi^{(0)}\left(\frac{\mu_{1}-\mu_{2}+\sqrt{c \Lambda}+t \sigma_{1} / \sqrt{n_{1}}}{\sigma_{2} / V \overline{n_{2}}}\right)-\Phi^{(0)}\left(\frac{\mu_{1}-\mu_{2}+\sqrt{c \bar{\lambda}}+t \sigma_{1} / \sqrt{n_{1}}}{\sigma_{2} / \sqrt{n_{2}}}\right)\right\} \\
& +\left(\frac{3 \boldsymbol{n}_{2}}{\sigma_{2}^{2}}+\frac{\boldsymbol{n}_{1}}{\sigma_{1}^{2}}\right) \frac{\sigma_{2}}{\sqrt{\boldsymbol{n}_{2}}}\left\{\Phi^{(1)}\left(\frac{\mu_{1}-\mu_{2}-\sqrt{\boldsymbol{c \lambda}}+t \sigma_{1} / \sqrt{\boldsymbol{n}_{1}}}{\sigma_{2} / \sqrt{\boldsymbol{n}_{2}}}\right)-\Phi^{(1)}\left(\frac{\mu_{1}-\mu_{2}-\sqrt{\boldsymbol{c} \Lambda}+t \sigma_{1} / \sqrt{\boldsymbol{n}_{1}}}{\sigma_{2} / \sqrt{\boldsymbol{n}_{2}}}\right)\right. \\
& \left.\left.+\Phi^{(1)}\left(\frac{\mu_{1} \mu_{2}+\sqrt{\boldsymbol{c \lambda}}+t \sigma_{1} / \sqrt{\boldsymbol{n}_{1}}}{\sigma_{2} / \sqrt{\boldsymbol{n}_{2}}}\right)-\Phi^{(1)}\left(\frac{\mu_{1}-\mu_{2}+\sqrt{\boldsymbol{c} \lambda}+t \sigma_{1} / \sqrt{\boldsymbol{n}_{1}}}{\sigma_{2} / V \overline{\boldsymbol{n}_{2}}}\right)\right\}\right] d t, \\
& \text { (2.3.5) M.S.D. }\{\bar{x}\}=\frac{1}{4}\left(\frac{\sigma_{1}^{2}}{n_{1}}+\frac{\sigma_{2}^{2}}{n_{2}}\right)-\frac{1}{\sqrt{2 \pi}} \int_{-\infty}^{\infty} e^{-t 2 / 2}\left(\frac { ( \frac { n _ { 1 } } { \sigma _ { 1 } ^ { 2 } } - \frac { n _ { 2 } } { \sigma _ { 2 } ^ { 2 } } ) } { 1 6 ( \frac { n _ { 1 } } { \sigma _ { 1 } ^ { 2 } } + \frac { n _ { 2 } } { \sigma _ { 2 } ^ { 2 } } ) ^ { 2 } \frac { n _ { 2 } } { \sigma _ { 2 } ^ { 2 } } } \left[\left(\frac{n_{1}}{\sigma_{1}^{2}}+\frac{7 n_{2}}{\sigma_{2}^{2}}\right)\right.\right. \\
& \cdot\left\{\Phi^{(2)}\left(\frac{\mu_{1}-\mu_{2}+\sqrt{\boldsymbol{c \lambda}}+t \sigma_{1} / \sqrt{\boldsymbol{n}_{1}}}{\sigma_{2} / \sqrt{\boldsymbol{n}_{2}}}\right)-\Phi^{(2)}\left(\frac{\mu_{1}-\mu_{2}-\sqrt{c \lambda}+t \sigma_{1} / \sqrt{\boldsymbol{n}_{1}}}{\sigma_{2} / \sqrt{\boldsymbol{n}_{2}}}\right)\right\} \\
& \left.-\left(\frac{5 n_{2}}{\sigma_{2}^{2}}+\frac{3 n_{1}}{\sigma_{1}^{2}}\right)\left\{\Phi^{(2)}\left(\frac{\mu_{1}-\mu_{2}+\sqrt{\boldsymbol{c} \Lambda}+t \sigma_{1} / \sqrt{n_{1}}}{\sigma_{2} / \sqrt{n_{2}}}\right)-\Phi^{(2)}\left(\frac{\mu_{1}-\mu_{2}-\sqrt{\boldsymbol{C} \Lambda}+t \sigma_{1} / \sqrt{n_{1}}}{\sigma_{2} / \sqrt{n_{2}}}\right)\right\}\right] \\
& -\frac{\left(\frac{n_{1}}{\sigma_{1}^{2}}-\frac{n_{2}}{\sigma_{2}^{2}}\right)}{8\left(\frac{\boldsymbol{n}_{1}}{\sigma_{1}^{2}}+\frac{\boldsymbol{n}_{2}}{\sigma_{2}^{2}}\right)^{2} \frac{\sqrt{\boldsymbol{n}_{2}}}{\sigma_{2}}}\left[\left\{\left(\frac{5 \boldsymbol{n}_{2}}{\sigma_{2}^{2}}-\frac{\boldsymbol{n}_{1}}{\sigma_{1}^{2}}\right)\left(\mu_{1}-\mu_{2}\right)-\frac{3 \sigma_{1}}{\sqrt{\boldsymbol{n}_{1}}}\left(\frac{\boldsymbol{n}_{1}}{\sigma_{1}^{2}}-\frac{\boldsymbol{n}_{2}}{\sigma_{2}^{2}}\right) t\right\}\left\{\stackrel{(1)}{\mu^{\mu_{1}}-\mu_{2}+\sqrt{\overline{c \lambda}}+t \sigma_{1} / \sqrt{\boldsymbol{n}_{1}}}\right)\right. \\
& \left.-\Phi^{(1)}\left(\frac{\mu_{1}-\mu_{2}-\sqrt{c \lambda}+t \sigma_{1} / \sqrt{\boldsymbol{n}_{1}}}{\sigma_{2} / \sqrt{\boldsymbol{n}_{2}}}\right)\right\}+\left\{\left(\frac{3 \boldsymbol{n}_{2}}{\sigma_{2}^{2}}+\frac{n_{1}}{\sigma_{1}^{2}}\right)\left(\mu_{1}-\mu_{2}\right)-\frac{\sigma_{1}}{\sqrt{\boldsymbol{n}_{1}}}\left(\frac{\boldsymbol{n}_{1}}{\sigma_{1}^{2}}-\frac{\boldsymbol{n}_{2}}{\sigma_{2}^{2}}\right) t\right\}
\end{aligned}
$$




$$
\begin{aligned}
& \left.\cdot\left\{\Phi^{(1)}\left(\frac{\mu_{1}-\mu_{2}+\sqrt{\boldsymbol{c} \Lambda}+t \sigma_{1} / \sqrt{\boldsymbol{n}_{1}}}{\sigma_{2} / \sqrt{\boldsymbol{n}_{2}}}\right)-\Phi^{(1)}\left(\frac{\mu_{1}-\mu_{2}-\sqrt{\boldsymbol{c} \Lambda}+t \sigma_{1} / \sqrt{\boldsymbol{n}_{1}}}{\sigma_{2} / \sqrt{\boldsymbol{n}_{2}}}\right)\right\}\right] \\
& -\frac{\left(\frac{\boldsymbol{n}_{1}}{\sigma_{1}^{2}}-\frac{\boldsymbol{n}_{2}}{\sigma_{2}^{2}}\right)}{16\left(\frac{\boldsymbol{n}_{1}}{\sigma_{1}^{2}}+\frac{\boldsymbol{n}_{2}}{\sigma_{2}^{2}}\right)^{2}}\left[\left\{3\left(\frac{\boldsymbol{n}_{1}}{\sigma_{1}^{2}}-\frac{\boldsymbol{n}_{2}}{\sigma_{2}^{2}}\right)\left(\mu_{1}-\mu_{2}\right)^{2}+\frac{2 \sigma_{1}}{\sqrt{\boldsymbol{n}_{1}}}\left(\frac{5 n_{1}}{\sigma_{1}^{2}}-\frac{n_{2}}{\sigma_{2}^{2}}\right)\left(\mu_{1}-\mu_{2}\right) t+\frac{\sigma_{1}^{2}}{\boldsymbol{n}_{1}}\left(\frac{7 n_{1}}{\sigma_{1}^{2}}+\frac{\boldsymbol{n}_{2}}{\sigma_{2}^{2}}\right) \boldsymbol{t}^{2}\right\}\right. \\
& \cdot\left\{\Phi^{(0)}\left(\frac{\mu_{1}-\mu_{2}+\sqrt{\boldsymbol{c} \lambda}+t \sigma_{1} / \sqrt{\boldsymbol{n}_{1}}}{\sigma_{2} / V \overline{n_{2}}}\right)-\Phi^{(0)}\left(\frac{\mu_{1}-\mu_{2}-\sqrt{\boldsymbol{c} \lambda}+t \sigma_{1} / V \overline{n_{1}}}{\sigma_{2} / \sqrt{\boldsymbol{n}_{2}}}\right)\right\} \\
& +\left\{\left(\frac{n_{1}}{\sigma_{1}^{2}}-\frac{n_{2}}{\sigma_{2}^{2}}\right)\left(\mu_{1}-\mu_{2}\right)^{2}+\frac{2 \sigma_{1}}{\sqrt{n_{1}}}\left(\frac{3 n_{1}}{\sigma_{1}^{2}}+\frac{n_{2}}{\sigma_{2}^{2}}\right)\left(\mu_{1}-\mu_{2}\right) t+\frac{\sigma_{1}^{2}}{n_{1}}\left(\frac{5 n_{1}}{\sigma_{1}^{2}}+\frac{3 n_{2}}{\sigma_{2}^{2}}\right) t^{2}\right\} \\
& \left.\cdot\left\{\Phi^{(0)}\left(\frac{\mu_{1}-\mu_{2}+\sqrt{\boldsymbol{c} \Lambda}+t \sigma_{1} / \sqrt{\boldsymbol{n}_{1}}}{\sigma_{2} / \overline{\boldsymbol{n}_{2}}}\right)-\Phi^{(0)}\left(\frac{\mu_{1}-\mu_{2}-\sqrt{\boldsymbol{c} \boldsymbol{\Lambda}}+t \sigma_{1} / \sqrt{\boldsymbol{n}_{1}}}{\sigma_{2} / \sqrt{\boldsymbol{n}_{2}}}\right)\right\}\right] d t,
\end{aligned}
$$

where we put that $\Phi^{(m)}(u)=(\sqrt{2 \pi})^{-1} \int_{-\infty}^{u} x^{m} \exp \left[-x^{2} / 2\right] d x$.

Proof: The mean value (2.3.4) is easily and the mean square deviation is obtained from the facts that

$$
\begin{aligned}
& E\left\{\overline{\bar{x}}^{2} \mid D_{(\mathrm{a})}\right\}=\left\{\sqrt{2 \pi}\left(\frac{n_{1}}{\sigma_{1}^{2}}+\frac{n_{2}}{\sigma_{2}^{2}}\right)^{2}\right\}^{-1} \int_{-\infty}^{\infty} e^{-t^{2} / 2}\left[\sum_{i=1}^{2}\left(\begin{array}{l}
2 \\
i
\end{array}\right)\left(\frac{n_{1}}{\sigma_{1}^{2}} \mu_{1}+\frac{n_{2}}{\sigma_{2}^{2}} \mu_{2}+\frac{\sqrt{n_{1}}}{\sigma_{1}} t\right)^{2-i}\right. \\
& \left.\cdot\left(\frac{\sqrt{\boldsymbol{n}_{2}}}{\sigma_{2}}\right)^{i}\left\{\Phi^{(i)}\left(\frac{\mu_{1}-\mu_{2}+V \overline{c \lambda}+t \sigma_{1} / \sqrt{\boldsymbol{n}_{1}}}{\sigma_{2} / V \overline{\boldsymbol{n}_{2}}}\right)-\Phi^{(i)}\left(\frac{\mu_{1}-\mu_{2}-\sqrt{\boldsymbol{c} \lambda}+t \sigma_{1} / \sqrt{\boldsymbol{n}_{1}}}{\sigma_{2} / V \overline{\boldsymbol{n}_{2}}}\right)\right\}\right] d t \text {, }
\end{aligned}
$$

$E\left\{\overline{\bar{x}}^{2} D_{(\mathrm{h})}\right\}=\left\{16 \sqrt{2 \pi}\left(\frac{n_{1}}{\sigma_{1}^{2}}+\frac{n_{2}}{\sigma_{2}^{2}}\right)^{2}\right\}^{-1} \int_{-\infty}^{\infty} e^{-t^{2} / 2}\left[\Sigma\left(\begin{array}{l}2 \\ i\end{array}\right)\left\{\left(\frac{3 n_{1}}{\sigma_{1}^{2}}+\frac{n_{2}}{\sigma_{2}^{2}}\right) \mu_{1}+\left(\frac{3 n_{2}}{\sigma_{2}^{2}}+\frac{n_{1}}{\sigma_{1}^{2}}\right) \mu_{2}\right.\right.$

$$
\begin{aligned}
& \left.+\frac{\sigma_{1}}{\sqrt{\boldsymbol{n}_{1}}}\left(\frac{3 \boldsymbol{n}_{1}}{\sigma_{1}^{2}}+\frac{\boldsymbol{n}_{2}}{\sigma_{2}^{2}}\right) \boldsymbol{t}\right\}^{2-i}\left\{\frac{\sigma_{2}}{V \overline{n_{2}}}\left(\frac{3 \boldsymbol{n}_{2}}{\sigma_{2}^{2}}+\frac{\boldsymbol{n}_{1}}{\sigma_{1}^{2}}\right)\right\}^{i}\left\{\Phi^{(i)}\left(\frac{\mu_{1}-\mu_{2}-\sqrt{\overline{c \lambda}}+t \sigma_{1} / \sqrt{\boldsymbol{n}_{1}}}{\sigma_{2} / \sqrt{n_{2}}}\right)\right. \\
& -\Phi^{(i)}\left(\frac{\mu_{1}-\mu_{2}-\sqrt{\boldsymbol{c} \boldsymbol{\Lambda}}+t \sigma_{1} / \sqrt{\boldsymbol{n}_{1}}}{\sigma_{2} / \sqrt{\boldsymbol{n}_{2}}}\right)+\Phi^{(i)}\left(\frac{\mu_{1}-\mu_{2}+\sqrt{\boldsymbol{c} \boldsymbol{\Lambda}}+t \sigma_{1} / \sqrt{\boldsymbol{n}_{1}}}{\sigma_{2} / \sqrt{\boldsymbol{n}_{2}}}\right) \\
& \left.\left.-\Phi^{(i)}\left(\frac{\mu_{1}-\mu_{2}+\sqrt{\boldsymbol{c} \bar{\lambda}}+t \sigma_{1} / \sqrt{\boldsymbol{n}_{1}}}{\sigma_{2} / \sqrt{\boldsymbol{n}_{2}}}\right)\right\}\right] d t,
\end{aligned}
$$

and

(2.3.8)E $\left\{x^{2} \mid D_{(\mathrm{c})}\right\}=\frac{\left(\mu_{1}+\mu_{2}\right)^{2}}{4}+\frac{1}{4}\left(\frac{\sigma_{1}^{2}}{n_{1}}+\frac{\sigma_{2}^{2}}{n_{2}}\right)-\frac{1}{4 \sqrt{2 \pi}} \int_{-\infty}^{-t^{2} / 2}\left[\sum_{i=0}^{2}\left(\begin{array}{l}2 \\ i\end{array}\right)\left(\mu_{1}+\mu_{2}+t \sigma_{1} / \sqrt{ } / \overline{n_{1}}\right)^{2-i}\right.$

$$
\left.\cdot\left(-\frac{\sigma_{2}}{\sqrt{n_{2}}}\right)^{i}\left\{\Phi^{(i)}\left(\frac{\mu_{1}-\mu_{2}+\sqrt{\boldsymbol{c} \Lambda}+t \sigma_{1} / \sqrt{\boldsymbol{n}_{1}}}{\sigma_{2} / \sqrt{\boldsymbol{n}_{2}}}\right)-\Phi^{(i)}\left(\frac{\mu_{1}-\mu_{2}-\sqrt{\boldsymbol{c} \Lambda}+t \sigma_{1} / V \overline{\boldsymbol{n}_{1}}}{\sigma_{2} / \sqrt{\boldsymbol{n}_{2}}}\right)\right\}\right] d t .
$$

Corollary 2.1 Specially when $\mu_{1}=\mu_{2}=\mu, \bar{x}$ is an unbiassed estimate of $\mu$ and M.S.D. $\{\overline{\bar{x}}\}$ becomes as follows: 


$$
\begin{aligned}
& \text { (2.3.9) M.S.D. }\{\overline{\bar{x}}\}=\frac{1}{4}\left(\frac{\sigma_{1}^{2}}{n_{1}}+\frac{\sigma_{2}^{2}}{n_{2}}\right)-\frac{1}{\sqrt{2 \pi}} \int_{-\infty}^{\infty} e^{-t^{2 / 2}} \frac{\frac{n_{1}}{\sigma_{1}^{2}}-\frac{n_{2}}{\sigma_{2}^{2}}}{16\left(\frac{n_{1}}{\sigma_{1}^{2}}+\frac{n_{2}}{\sigma_{2}^{2}}\right)^{2}}\left[\left(\frac{n_{1} \sigma_{1}^{2}}{n_{2} \sigma_{2}^{2}}+7\right)\right.
\end{aligned}
$$

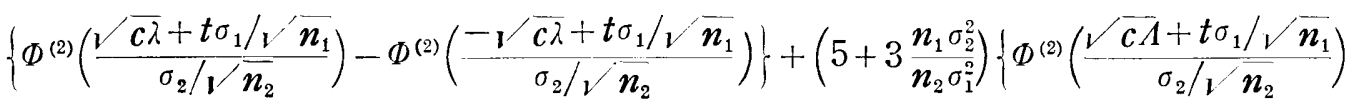

$$
\begin{aligned}
& \left.\left.-\Phi^{(2)}\left(\frac{-\sqrt{\boldsymbol{c} \Lambda}+t \sigma_{1} / \sqrt{\boldsymbol{n}_{1}}}{\sigma_{2} / \sqrt{\boldsymbol{n}_{2}}}\right)\right\}\right]+\frac{\left(\frac{n_{1}}{\sigma_{1}^{2}}-\frac{\boldsymbol{n}_{2}}{\sigma_{2}^{2}}\right)}{8\left(\frac{\boldsymbol{n}_{1}}{\sigma_{1}^{2}}+\frac{\boldsymbol{n}_{2}}{\sigma_{2}^{2}}\right)} \frac{\sigma_{1} \sigma_{2}}{\sqrt{\boldsymbol{n}_{1} \boldsymbol{n}_{2}}} t\left[3 \left\{\Phi^{(1)}\left(\frac{\sqrt{\boldsymbol{c \lambda}}+t \sigma_{1} / \sqrt{\boldsymbol{n}_{1}}}{\sigma_{2} / \sqrt{\boldsymbol{n}_{2}}}\right)\right.\right. \\
& \left.\left.-\Phi^{(1)}\left(\frac{-\sqrt{\boldsymbol{c} \lambda}+t \sigma_{1} / \sqrt{\boldsymbol{n}_{1}}}{\sigma_{2} / \sqrt{\boldsymbol{n}_{2}}}\right)\right\}+\Phi^{(1)}\left(\frac{\sqrt{\boldsymbol{c} \Lambda}+t \sigma_{1} / \sqrt{\boldsymbol{n}_{1}}}{\sigma_{2} / \sqrt{\boldsymbol{n}_{2}}}\right)-\Phi^{(1)}\left(\frac{-\sqrt{\boldsymbol{c} \Lambda}+t \sigma_{1} / \sqrt{\boldsymbol{n}_{1}}}{\sigma_{2} / v \boldsymbol{n}_{2}}\right)\right] \\
& -\frac{\left(\frac{\boldsymbol{n}_{1}}{\sigma_{1}^{2}}-\frac{\boldsymbol{n}_{2}}{\sigma_{2}^{2}}\right)}{16\left(\frac{\boldsymbol{n}_{1}}{\sigma_{1}^{2}}+\frac{\boldsymbol{n}_{2}}{\sigma_{2}^{2}}\right)^{2}} t^{2}\left[\left(7+\frac{\boldsymbol{n}_{2} \sigma_{1}^{2}}{\boldsymbol{n}_{1} \sigma_{2}^{2}}\right)\left\{\Phi^{(0)}\left(\frac{\sqrt{c \lambda}+t \sigma_{1} / \sqrt{\boldsymbol{n}_{1}}}{\sigma_{2} / \sqrt{\boldsymbol{n}_{2}}}\right)-\Phi^{(0)}\left(\frac{-\sqrt{c \lambda}+t \sigma_{1} / \sqrt{\boldsymbol{n}_{1}}}{\sigma_{2} / \mathrm{v} / \overline{\boldsymbol{n}_{2}}}\right)\right\}\right. \\
& \left.\left.+\left(5+3 \frac{\boldsymbol{n}_{2} \sigma_{1}^{2}}{\boldsymbol{n}_{1} \sigma_{2}^{2}}\right)\left\{\Phi^{(0)}\left(\frac{\sqrt{\boldsymbol{c} \Lambda}+t \sigma_{1} / V \overline{\boldsymbol{n}_{1}}}{\sigma_{2} / \sqrt{\boldsymbol{n}_{2}}}\right)-\Phi^{(0)}\left(\frac{-\sqrt{\boldsymbol{c} \Lambda}+t \sigma_{1} / \sqrt{\boldsymbol{n}_{1}}}{\sigma_{\bar{z}} / \sqrt{\boldsymbol{n}_{2}}}\right)\right\}\right]\right) d t .
\end{aligned}
$$

Corollary 2.2. Specially the inference procedure defined by $\Lambda=\lambda=0$ shows us that $E\{\overline{\bar{x}}\}=\left(\mu_{1}+\mu_{2}\right) / 2$ and M.S.D. $\{\overline{\bar{x}}\}=\left(\frac{\sigma_{1}^{2}}{n_{1}}+\frac{\sigma_{2}^{2}}{n_{2}}\right) / 4$, while that defined by $A=\lambda=\infty$ shows us that $E\{\overline{\bar{x}}\}=\left(\frac{n_{1}}{\sigma_{1}^{2}} \mu_{1}+\frac{n_{2}}{\sigma_{2}^{2}} \mu_{2}\right) /\left(\frac{n_{1}}{\sigma_{1}^{2}}+\frac{n_{2}}{\sigma_{2}^{2}}\right)$ and $M . S$. $D .\{\bar{x}\}=\left(\frac{n_{1}}{\sigma_{1}^{2}}+\frac{n_{2}}{\sigma_{2}^{2}}\right)^{-1}+\left(\frac{n_{1}}{\sigma_{1}^{2}}-\frac{n_{2}}{\sigma_{2}^{2}}\right)^{2}\left(\mu_{1}-\mu_{2}\right)^{2} / 4\left(\frac{n_{1}}{\sigma_{1}^{2}}+\frac{n_{2}}{\sigma_{2}^{2}}\right)^{2}$.

§3. The inferences using the results of two biological assays with unknown variances under the assumption of an equality of population means

When the potency of a test preparation is estimated in the analytical assay of twice or more times, their results may be considered occasionally that good agreement between several estimates is to expect. In these circumstances of the biological assay, the combined estimate is used to take a weighted mean with weights inversely proportional to the individual variances and knowing the values of their variances we obtain a best estimate by the statistic. But for such an explicit information concerning to the values of the individual variances, we may have no choice except for substituting the estimates of their variances and we are confronted mostly with lack of the information in practice. For instance, we may be able to illustrate a potency estimation for a tuberculin preparation, one by assay on cows and one on guineapigs, Fisher [1], Finney [1]. 
Now let $O_{n_{1}}:\left(x_{11}, x_{12}, \ldots, x_{1 n_{1}}\right)$ be a random sample of $n_{1}$ from a normal population $N\left(\mu, \sigma_{1}^{2}\right)$ and let $O_{n_{2}}:\left(x_{21}, x_{22}, \ldots, x_{2 n_{2}}\right)$ be a random sample of $n_{2}$ from another normal population $N\left(\mu, \sigma_{2}^{2}\right)$. The population variances $\sigma_{1}^{2}$ and $\sigma_{2}^{2}$ may be or may not be distinct.

Then our rule of inference procedure is defined in the following way:

(i) Let $\bar{x}_{i}$ and $s_{i}^{2}$ be the sample mean and the sample unbiassed variance from $O_{n i},(i=1,2)$.

(ii) Let the statistic $F$ be defined by $F=s_{2}^{2} / s_{1}^{2}$.

(iii) Now let us introduce the estimate $\overline{\bar{x}}$ of $\mu$ defined in the following way:

(a) $\overline{\bar{x}}=\left(n_{1} \bar{x}_{1}+n_{2} \bar{x}_{2}\right) /\left(n_{1}+n_{2}\right)$, if $F \leq \Lambda$,

(b) $\overline{\bar{x}}=\left(w_{1} \bar{x}_{1}+w_{2} \bar{x}_{2}\right) /\left(w_{1}+w_{2}\right)$, if $F>\Lambda$,

where $w_{i}=n_{i} / s_{i}^{2},(i=1,2)$, and the switching constant $A$ is prescribed constant.

Our attitude of the successive inference is asserted in the following way: We may prefer to take the weighted mean $\left(w_{1} \bar{x}_{1}+w_{2} \bar{x}_{2}\right) /\left(w_{1}+w_{2}\right)$ as a substitution of a best estimate of $\mu$ in case when a distinction between the values of two sample unbiassed variances $\hat{\sigma}_{1}^{2}$ and $\hat{\sigma}_{2}^{2}$ is shown significantly under a hypothesis $H: \sigma_{1}^{2}=\sigma_{2}^{2}$, and otherwise we may take naturally $\left(n_{1} \bar{x}_{1}+\right.$ $\left.n_{2} \bar{x}_{2}\right) /\left(n_{1}+n_{2}\right)$ as a best estimate under the assumption of the same population in case when an equality in the hypothesis $H$ has nothing to be denied from the test of sample variances and at the same time we shall be much relieved from the troublesome calculation of the weighted mean.

The another aspect of the attitude of our inference procedure may be explained from a different view-point of an assumption $\sigma_{1}^{2}<\sigma_{2}^{2}$ given by the information concerning to the population variances, and in principle we may consider now to adopt always the weighted mean with weights inversely proportional to the individual variances. Under the circumstances of the observations, however, if $s_{2}^{2}$ happens to be smaller significantly than $s_{1}^{2}$ in case that $F<A$, we may consider to be unable to admit the dispersion of observations with some unreliability and may prefer to take the sample means merely which are independent of the dispersions statistically and to combine by the formation of (a). That is to say, we come to the conclusion that adopting the weights which is contrary to the assumption is inadequately and we may and now shall introduce the successive inference with certain validity check of the observations by the preliminary testing procedure.

Theorem 3.1. The distribution function of $\overline{\bar{x}}$ is given by

$$
\begin{gathered}
\operatorname{Pr} .\{\bar{x}<u\}=\operatorname{Pr} .\left\{\left(n_{1} \bar{x}_{1}+n_{2} \bar{x}_{2}\right) /\left(n_{1}+n_{2}\right)<u, D_{(u)}\right\}+\operatorname{Pr} .\left\{\left(w_{1} \bar{x}_{1}+w_{2} \bar{x}_{2}\right) /\left(w_{1}\right.\right. \\
\left.\left.+w_{2}\right)<u, D_{(b)}\right\}=\Phi\left(\frac{2 \sqrt{n_{1} n_{2}}(u-\mu)}{\sqrt{n_{2} \sigma_{1}^{2}+n_{1} \sigma_{2}^{2}}}\right) \cdot I_{\tau}\left(\frac{\nu_{2}}{2}, \frac{\nu_{1}}{L}\right)+\iint_{D}\left\{\int_{\Lambda \sigma_{1}^{2} / \sigma_{2}^{2}}^{\infty} f\left(\bar{x}_{1}, \bar{x}_{2}, G\right) d G\right\} \\
\cdot d \bar{x}_{1} d \bar{x}_{2},
\end{gathered}
$$


where $D_{(\text {a) }}$ is the domain defined in the sample space $\left(x_{11}, \ldots, x_{1 n_{1}}, x_{21}, \ldots\right.$, $x_{2 n_{2}}$ ) by the above relation $(a)$ and $D_{(b)}$ is the complement set of $D_{(\mathrm{a})}$ in the same space, and where

$$
\left\{\begin{array}{l}
I_{\tau}\left(\frac{\nu_{2}}{2}, \frac{\nu_{1}}{2}\right)=B_{\tau}\left(\frac{\nu_{2}}{2}, \frac{\nu_{1}}{2}\right) / B\left(\frac{\nu_{2}}{2}, \frac{\nu_{1}}{2}\right), \tau=\nu_{2} \sigma_{1}^{2} \Lambda /\left(\nu_{2} \sigma_{1}^{2} \Lambda+\nu_{1} \sigma_{2}^{2}\right), \\
f\left(\bar{x}_{1}, \bar{x}_{2}, G\right)=\frac{\sqrt{n_{1} n_{2}}}{2 \pi \sigma_{1} \sigma_{2}} \frac{\left(\nu_{2} / \nu_{1}\right)^{\nu_{2} / 2}}{B\left(\frac{\nu_{1}}{2}, \frac{\nu_{2}}{2}\right)} G^{\frac{\nu_{2}}{2}-1}\left(1+\frac{\nu_{2}}{\nu_{1}} G\right)^{-\frac{\nu_{1}+\nu_{2}}{2}} \exp \left[-\frac{1}{2}\left\{\frac{n_{1}\left(\bar{x}_{1}-\mu\right)}{\sigma_{1}^{2}}\right.\right. \\
\left.\left.+\frac{n_{2}\left(\bar{x}_{2}-\mu\right)}{\sigma_{2}^{2}}\right\}\right], \mathfrak{D}:-\infty<\frac{c_{1} G \bar{x}_{1}+\bar{x}_{2}}{1+c_{1} G}<u, c_{1}=n_{1} \sigma_{2}^{2} / n_{2} \sigma_{1}^{2}, \nu_{i}=n_{i}-1 .
\end{array}\right.
$$

Proof: The proof is immediate and shall be omitted.

Theorem 3.2. The mean value $E\{\overline{\bar{x}}\}$ and the mean square deviation $E\left\{(\overline{\bar{x}}-\mu)^{2}\right\}$ of the estimate $\overline{\bar{x}}$ are given by

(3.3) $E\{\bar{x}\}=\mu$,

and

$$
\text { M.S.D. }\{\overline{\bar{x}}\}=\frac{n_{1} \sigma_{1}^{2}+n_{2} \sigma_{2}^{2}}{\left(n_{1}+n_{2}\right)^{2}}\left\{1-I_{\theta}\left(\frac{\nu_{1}}{2}, \frac{\nu_{2}}{2}\right)\right\}+\frac{\sigma_{2}^{2}}{n_{2}}\left\{\left(\frac{\nu_{1}}{\nu_{2}}\right)^{2} c_{1} J_{1}+J_{2}\right\}
$$

where

$$
\begin{aligned}
J_{1} & =\frac{1}{B\left(\frac{\nu_{1}}{2}, \frac{\nu_{2}}{2}\right)} \sum_{i=0}^{\infty}(i+1)\left(1-\frac{1}{T}\right)^{i} B_{\theta}\left(\frac{\nu_{1}}{2}, \frac{\nu_{2}}{2}+i+2\right) & \text { for } T \neq 1, \\
& =B_{\theta}\left(\frac{\nu_{1}}{2}, \frac{\nu_{2}}{2}+2\right) / B\left(\frac{\nu_{1}}{2}, \frac{\nu_{2}}{2}\right), & \text { for } T=1, \\
J_{2} & =\frac{1}{B\left(\frac{\nu_{1}}{2}, \frac{\nu_{2}}{2}\right)} \sum_{i=0}^{\infty}(i+1)\left(1-\frac{1}{T}\right)^{i} B_{\theta}\left(\frac{\nu_{1}}{2}+2, \frac{\nu_{2}}{2}+i\right) & \text { for } T \neq 1, \\
& =B_{\theta}\left(\frac{\nu_{1}}{2}+2, \frac{\nu_{2}}{2}\right) / B\left(\frac{\nu_{1}}{2}, \frac{\nu_{2}}{2}\right), & \text { for } T=1,
\end{aligned}
$$

and

$$
\theta=\nu_{1} \sigma_{2}^{2} /\left(\nu_{2} \sigma_{1}^{2} \Lambda+\nu_{1} \sigma_{2}^{2}\right), T=n_{2} \nu_{2} \sigma_{1}^{2} / n_{1} \nu_{1} \sigma_{2}^{2} .
$$

Proof : The mean value (3.3) is obtined immediately, and the mean square deviation is obtained by

$$
E\{\overline{\bar{x}}\}=E\left\{\left(\frac{c_{1} \bar{x}_{1} G+\bar{x}_{2}}{1+c_{1} G}\right)^{2} \mid D_{(\mathrm{a})}\right\}+E\left\{\left(\frac{n_{1} \bar{x}_{1}+n_{2} \bar{x}_{2}}{n_{1}+n_{2}}\right)^{2} \mid D_{(1))}\right\},
$$

where 


$$
E\left\{\left(\frac{c_{1} \bar{x}_{1} G+\bar{x}_{2}}{1+c_{1} G}\right)^{2} D_{(:)}\right\}=\mu^{2}\left\{1-I_{z}\left(\frac{\nu_{2}}{2}, \frac{\nu_{1}}{2}\right)\right\}+\frac{\sigma_{1}^{2} c_{1}^{2}}{n_{1}}\left(\frac{\nu_{1}}{\nu_{2}}\right)^{2} J_{1}+\frac{\sigma_{2}^{2}}{n_{2}} J_{2}
$$

and

$$
E\left\{\left(\frac{n_{1} \bar{x}_{1}+n_{2} \bar{x}_{2}}{n_{1}+n_{2}}\right)^{2} \mid D_{(\mathrm{b})}\right\}=\left\{\mu^{2}+\frac{n_{1} \sigma_{1}^{2}+n_{2} \sigma_{2}^{2}}{\left(n_{1}+n_{2}\right)^{2}}\right\} I_{\tau}\left(\frac{\nu_{2}}{2}, \frac{\nu_{1}}{2}\right)
$$

and the convergency of each $J$ of (3.5) may be easily shown in view of the situation of the respective original integrations regardless of any value of $T$.

Corollary 3.1. Specially when $\sigma_{1}^{2}=\sigma_{2}^{2}=\sigma^{2}$ and we take that $n_{1}=n_{2}=n$, the estimate $\overline{\bar{x}}$ is constantly an unbiassed estimate of $\mu$ and M.S.D. $\{\overline{\bar{x}}\}$ becomes as follows:

$$
\text { M.S.D. }\{\overline{\bar{x}}\}=\frac{\sigma^{2}}{2 n}\left[1-I_{\theta_{0}}\left(\frac{\nu}{2}, \frac{\nu}{2}\right)+\frac{n+1}{2 n}\left\{I_{\theta_{0}}\left(\frac{\nu}{2}, \frac{\nu}{2}+2\right)+I_{\theta_{0}}\left(\frac{\nu}{2}+2, \frac{\nu}{2}\right)\right\}\right] \text {, }
$$

where $\theta_{0}=(\Lambda+1)^{-1}$ and $\nu=n-1$. Moreover if we put $\Lambda=0$,

$$
\text { M.S.D. }\{\overline{\bar{x}}\}=\frac{n+1}{2 n^{2}} \sigma^{2},
$$

and otherwise if we put $A=\infty, M . S . D .\{\bar{x}\}=\sigma^{2} / 2 n$. And in this special case we may find easily that taking $\Lambda=\infty$ is prefered.

Corollary 3.2. Specially an inference procedure defined by gives us an unbiassed estimate of $\mu$ and M.S.D. $\{\overline{\bar{x}}\}=\left(n_{1} \sigma_{1}^{2}+n_{2} \sigma_{2}^{2}\right) /\left(n_{1}+n_{2}\right)^{2}$. Wrile, the defined by $\Lambda=0$ gives us the unbiassed estimate and M.S.D. $\{\overline{\bar{x}}\}=\left\{c_{1}\left(\frac{\nu_{1}}{\nu_{2}}\right)^{2} J_{1}+J_{2}\right\} \frac{\sigma_{2}^{2}}{n_{2}}$.

§ 4. The inference using the results of two biological assays with unknown common variances in case when the equality of population means is not assumed

The inference procedure discussed here is almost corresponding to the previous procedure defined by $\S 2.1$ except for that the values of two population variances are common and are unknown to us. That is to say, the potency of a test preparation is estimated through a preliminary test of the hypothesis on equality of the population means on the basis of the similar motivation as $\$ 2.1$ for the improvement of the precision of estimate.

Now let $O_{n_{1}}:\left(x_{11}, x_{12}, \ldots, x_{1 n_{1}}\right)$ be a random sample of $n_{1}$ from a normal population $N\left(\mu_{1}, \sigma^{2}\right)$ and let $O_{n_{2}}:\left(x_{21}, x_{22}, \ldots, x_{2 n_{2}}\right)$ be a random sample of $n_{2}$ from another normal population $N\left(\mu_{2}, \sigma^{2}\right)$, where the population may be or may not be distinct, but an equality of the population variances is assumed.

Then our rule of inference procedure is defined in the following way:

(i) Let $\bar{x}_{i}$ and $s_{i}^{2}$ be the sample mean and the sample unbiassed vari- 
ance from $O_{n_{i}},(i=1,2)$,

(ii) Let the statistic $t$ be defined by

$$
t=\frac{\bar{x}_{1}-\bar{x}_{2}}{\sqrt{\left(n_{1}-1\right) s_{1}^{2}+\left(n_{2}-1\right) s_{2}^{2}}} \cdot \frac{\left.\sqrt{n_{1} n_{2}\left(n_{1}+n_{2}\right.}-2\right)}{\sqrt{n_{1}+n_{2}}} .
$$

(iii) Now let us introduce the estimate $\bar{x}$ of $\left(\mu_{1}+\mu_{2}\right) / 2$ defined in the following way:

(a) $\overline{\bar{x}}=\left(n_{1} \bar{x}_{1}+\boldsymbol{n}_{2} \bar{x}_{2}\right) /\left(n_{1}+n_{2}\right)$, if $|t| \leq \Lambda$,

(b) $\bar{x}=\left(\bar{x}_{1}+\bar{x}_{2}\right) / 2$, if $|t|>\Lambda$,

where the constant $A$ is prescribed and is corresponding to a value of $\alpha_{\Lambda^{-}}$ point of the $\boldsymbol{t}$-distribution with degrees of freedom $\left(\boldsymbol{n}_{1}+\boldsymbol{n}_{2}-2\right)$.

Here we may note the present attitude of the inference procedure in the following way: When a significance between two population means is shown us by a fact $|t|>A$, we may prefer to make an unbiassed estimate $\left(\bar{x}_{1}+\bar{x}_{2}\right) / 2$ in order to estimate a value of $\left(\mu_{1}+\mu_{2}\right) / 2$, and otherwise when the significance is not recognized by the fact $|t| \leq \Lambda$, that is to say, when the hypothesis that $\mu_{1}=\mu_{2}$ is unable to be rejected, we may and now shall accept the hypothesis and take the weighted mean to aim at a best estimate of common $\mu\left(=\mu_{1}=\mu_{2}\right)$, where the weighted mean is immediately given by $\left(n_{1} \bar{x}_{1}+n_{2} \bar{x}_{2}\right) /\left(n_{1}+n_{2}\right)$, instead of $\left(w_{1} \bar{x}_{1}+w_{2} \bar{x}_{2}\right) /\left(w_{1}+w_{2}\right)$, from the view-point of that the weights of $\bar{x}_{1}$ and $\bar{x}_{2}$ are only denoted in proportion to $n_{1}$ and $n_{2}$ under the prescribed assumption of the equality of the population variances. dure.

We are now going some results given by the present inference proce-

Theorem 4.1. The distribution function of $\overline{\bar{x}}$ is given by

$$
\begin{aligned}
& \operatorname{Pr} .\{\bar{x}<u\}=\frac{1}{\sqrt{2 \pi}} \Phi\left(\frac{\left(n_{1}+n_{2}\right) u-\left(n_{1} \mu_{1}+n_{2} \mu_{2}\right)}{\sigma \sqrt{n_{1}+n_{2}}}\right) \iint_{T S \leq \Lambda} e^{-\frac{p^{2}}{2}} \frac{V^{\frac{n_{1}+n_{2}}{2}-2} e^{-V}}{\Gamma\left(\frac{n_{1}+n_{2}-2}{2}\right)} d p d V \\
& +\frac{1}{\sqrt{2 \pi}} \int_{|T|>\Lambda} \Phi\left(\left(u-\frac{\mu_{1}+\mu_{2}}{2}\right) \frac{2 \sqrt{n_{1}+n_{2}}}{\sigma}-\frac{n_{1}-n_{2}}{\sqrt{n_{1} n_{2}}} p\right) e^{-\frac{p^{2}}{2}} \frac{V^{\frac{n_{1}+n_{2}}{2}-2} e^{-V}}{\Gamma\left(\frac{n_{1}+n_{2}-2}{2}\right)} d p d V,
\end{aligned}
$$

where

$$
T=\left(\sqrt{\frac{\boldsymbol{n}_{1}+\boldsymbol{n}_{2}}{\boldsymbol{n}_{1} \boldsymbol{n}_{2}}} \frac{\mu_{2}-\mu_{1}}{\sigma}+p\right) / v / \overline{2 V /\left(\boldsymbol{n}_{1}+n_{2}-2\right)} .
$$

Theorem 4.2. The mean value $E\{\overline{\bar{x}}\}$ and the mean square deviation M.S.D. $\{\overline{\bar{x}}\}$ are given by

$$
E\{\bar{x}\}=\frac{\mu_{1}+\mu_{2}}{2}+\frac{\left(n_{1}-n_{2}\right)\left(\mu_{1}-\mu_{2}\right)}{2\left(n_{1}+n_{2}\right)} \int_{|Y| \leq \Lambda} f(p, V) d p d V+\frac{\left(n_{1}-n_{2}\right) \sigma}{2 \sqrt{n_{1} n_{2}\left(n_{1}+n_{2}\right)}}
$$




$$
\iint_{|T|>\Lambda} p f(p, V) d p d V
$$

and

$$
\begin{array}{r}
M . S . D .\{\overline{\bar{x}}\}=\frac{\sigma^{2}}{n_{1}+n_{2}}+\frac{\left(n_{1}-n_{2}\right)^{2}\left(\mu_{1}-\mu_{2}\right)^{2}}{4\left(n_{1}+n_{2}\right)^{2}} \int_{\mid T \leq \Lambda} f(p, V) d p d V \\
\quad+\frac{\left(n_{1}-n_{2}\right)^{2} \sigma^{2}}{4 \cdot n_{1} n_{2}\left(n_{1}+n_{2}\right)} \int_{T ;>\Lambda} p_{\Lambda} f(p, V) d p d V,
\end{array}
$$

where we put that $f(p, V)=V^{\frac{n_{1}+n_{2}}{2}-2} e^{-\left(\frac{p^{2}}{2}+V\right)} / \sqrt{2 \pi} \Gamma\left(\frac{n_{1}+n_{2}-2}{2}\right)$.

Corollary 4.1. Specially the inference procedure defined by $A=\infty$ gives us that $E\{\bar{x}\}=\left(\mu_{1}+\mu_{2}\right) / 2+$ "bias", where the "bias" is $\left(n_{1}-n_{2}\right)\left(\mu_{1}\right.$ $\left.-\mu_{2}\right) / 2\left(n_{1}+n_{2}\right)$, and M.S.D. $\{\overline{\bar{x}}\}=\sigma^{2} /\left(n_{1}+n_{2}\right)+\left(n_{1}-n_{2}\right)^{2}\left(\mu_{1}-\mu_{2}\right)^{2} / 4 \cdot\left(n_{1}+n_{2}\right)^{2}$. While, the defined by $\Lambda=0$ gives us an unbiassed estimate of $\left(\mu_{1}+\mu_{2}\right) / 2$, but the M.S.D. $\{\bar{x}\}$ becomes $\left(n_{1}+n_{2}\right) \sigma^{2} / 4 n_{1} n_{2}$ and does not always give a minimum value of the M.S.D. $\{\overline{\bar{x}}\}$. Hence certain choice of a proper value of $\Lambda$ may be prefered in view of minimizing the mean square deviation (4.3).

Corollary 4.2. Specially when $\mu_{1}=\mu_{2}=\mu, \overline{\bar{x}}$ is an unbiassed estimate of $\mu$ and the M.S.D. $\{\overline{\bar{x}}\}$ becomes as follows:

$$
\text { M.S.D. }\{\overline{\bar{x}}\}=\frac{\sigma^{2}}{n_{1}+n_{2}}\left\{1+\frac{\left(n_{1}-n_{2}\right)^{2}}{4 n_{1} n_{2}} \int_{\{T \mid>\Lambda} p^{2} f(p, V) d p d V\right\}
$$

And we may find easily that the inference defined by $\Lambda=\infty$ is prefered and then we obtain the best estimate of $\mu$.

\section{Further discussion and conclusion}

In this paper, the inference procedures concerning the combination of estimates from two different biological assays are discussed and are given certain properties caused by the respective inference rule, in order to aim at the improvement of the precision of an estimate of the potency.

The only concerns in this paper, like in Asano [1], may be to choose the switching constants prescribed by $\lambda$ and/or $\Lambda$. And, in the sense of ordinary testing hypothesis of certain validity test of observations, most biometricians in practice may choose the values of 95 or 99 percent-point of other percent-point of a basic distribution of certain statistic to be tested for the sake of the consideration of the probabilities of 1 st and 2 nd kind error like in Tang [1] or Lehmer [1] as the values of $\lambda$ and/or $A$. But the values of $\lambda$ and/or $A$ have influence upon the accuracy and the precision of the estimate and hence may be considered to employ such certain values of $\lambda$ and/or $\Lambda$ as will minimize a maximum mean square deviation. 
In conclusion, in order to know the numerical appreciation of the inference procedure in the sense of ordinary testing hypothesis and moreover to find the values of $\lambda$ and/or $A$ in the sense of certain game-theoretical strategies, the numerical tables for the respective inference rule are quite important and now are going to be prepared by the author. KYUSHU UNIVERSITY and SHIONOGI PHARMACEUTICAL CO.

\section{References}

Asano, C. [1] : Estimations after preliminary test of significance and their applications to biometrical researches, Bull. Math. Stat., 9-2 (1960), 1-23.

Asano, C. and Sugimura, M. [1] : Some considerations on estimation of population variance due to the use of pooling data, Bull. Math. Stat., 9-3,4 (1960), 33-45.

Bancroft, T. A. [1] : On biases in estimation due to the use of preliminary tests of significance. Ann. Math. Stat., 15 (1944), 190-204.

Bennet, C. I. [1] : Estimation of means on the basis of preliminary tests of significance, Ann. Inst, Stat. Math., Tokyo, 4 (1952), 31-42.

Bliss, C. I. [1] : The statistics of bioassay, Academic Press, (1952).

Cochran, W. G. [1] : Problems arising in the analysis of a series of similar experiments, Jour. Roy. Stat. Soc., Supp 4 (1937), 102-18.

Cochran, W. G. [2] : The combination of estimates from different experiments, 10 (1954), 10129.

Finney, D. J. [1] : Statistical method in biological assay, Charles Griffin \& Co., (1952).

Fisher, R. A. [1] : A biological assay of tuberculins, Biometrics, 5 (1949), 300-16.

Huntsberger, D. V. [1] : A generalization of a preliminary testing procedure for pooling data, Ann. Math Stat., 26 (1955), 734-43.

Kitagawa, T. [1] : Successive process of statistical inferences, Mem. Fac. of Sci. Kyushu Univ., Ser. A, 5 (1950), 139-80.

[2]: Successive process of statistical inferences (5), Mem. Fac. of Sci. Kyushu Univ., Ser. A, 7 (1953), 81-106.

Lehmer, E. [1] : Tables of required for a given power, inverse tables of probabilities of errors of the second kind, Ann. Math. Stat. 15 (1944), 388-98.

Tang, P. C. [1] : The power function of the analysis of variance test with tables and illustrations of their use, Stat. Res. Mem., 2 (1938), 126-57.

Yates, F. and Cochran, W. G. [1] : The analysis of groups of experiments, Jour. Agr. Sci., 28 (1938), 556-80. 